\title{
Structural neuroanatomy of tinnitus and hyperacusis in semantic dementia
}

\author{
Colin J Mahoney, Jonathan D Rohrer, Johanna C Goll, Nick C Fox, Martin N Rossor, \\ Jason D Warren
}

Dementia Research Centre, Institute of Neurology, University College London, London, UK

\section{Correspondence to} Dr J D Warren, Dementia Research Centre, Institute of Neurology, 8-11 Queen Square, London WC1N 3BG, UK; jwarren@drc.ion.ucl.ac.uk

Received 16 November 2010 Revised 3 March 2011 Accepted 24 March 2011 Published Online First 28 April 2011

\section{Q) UN OCKKA}

This paper is freely available online under the BMJ Journals unlocked scheme, see http:// jnnp.bmj.com/site/about/ unlocked.xhtml

\begin{abstract}
Introduction Tinnitus and hyperacusis are common symptoms of excessive auditory perception in the general population; however, their anatomical substrates and disease associations continue to be defined. Patients with semantic dementia (SemD) frequently report tinnitus and hyperacusis but the significance and basis for these symptoms have not been elucidated.

Methods 43 patients with a diagnosis of SemD attending a specialist cognitive disorders clinic were retrospectively studied. 14 patients (32\% of the cohort) reported at least moderately severe chronic auditory symptoms: seven had tinnitus and a further seven had hyperacusis, and all had brain MRI while symptomatic. MRI data from SemD patients with and without auditory symptoms were compared using voxel based morphometry in order to identify neuroanatomical associations of tinnitus and hyperacusis.

Results Compared with SemD patients with no history of auditory symptoms, patients with tinnitus or hyperacusis had relative preservation of grey matter in the posterior superior temporal lobe and reduced grey matter in the orbitofrontal cortex and medial geniculate nucleus.

Conclusions Tinnitus and hyperacusis may be a significant issue in SemD. Neuroanatomical evidence in SemD supports previous work implicating a distributed cortico-subcortical auditory and limbic network in the pathogenesis of these abnormal auditory percepts.
\end{abstract}

\section{INTRODUCTION}

Tinnitus and hyperacusis are common symptoms of altered and excessive auditory perception. ${ }^{1}{ }^{2}$ Tinnitus may be defined as abnormal perception of (elementary) sound in the absence of an external source. It is usually experienced as 'buzzing' or 'ringing', distinguishing it from more organised auditory hallucinations. The reported overall prevalence of tinnitus in the general population in a large postal study was $10 \%{ }^{2}$ Tinnitus becomes more prevalent in association with ageing and hearing loss, with an estimated prevalence of $12-18 \%$ over the age of 60 years $^{2}{ }^{3}$; however, in only a minority of these individuals is it a significant clinical issue. Tinnitus severity has been quantified and its impact on sufferers' daily lives has been assessed using questionnaires such as the Tinnitus Handicap Inventory (THI). ${ }^{4}$ The pathogenesis of tinnitus remains poorly understood ${ }^{5} 6$ and although most often associated with otological disease, both peripheral and central factors are likely to play a role. Neuroanatomical associations for tinnitus have been studied using structural and functional brain imaging techniques (summarised in table 1) but have yet to be fully defined. Many brain regions and structures, including auditory cortex, thalamus, inferior colliculus, limbic and paralimbic areas have been implicated. ${ }^{7-12}$ One influential model of tinnitus pathogenesis posits an interaction between hyperexcitable peripheral auditory neurons and underactive modulating or inhibitory central neurons, with a net emergence of abnormal autonomous activity in central circuits, experienced as an abnormal percept. ${ }^{5}$

Hyperacusis may be defined as reduced tolerance of sound often leading to avoidance of noisy environments. ${ }^{13}$ The prevalence of chronic hyperacusis in the general population aged 51-79 years has been estimated as up to $9 \%$ using a postal survey. ${ }^{1}$ Hyperacusis has been less well studied than tinnitus but these symptoms frequently co-occur, and shared pathophysiological mechanisms have been proposed..$^{13} 14$ The most common association of hyperacusis is migraine but a range of other disease associations are recognised. ${ }^{13}$ It occurs in up to $90 \%$ of patients with Williams syndrome ${ }^{14}$ in whom it appears to be part of a broader auditory phenotype with altered responsivity to sound; disturbed metabolism of 5-hydoxytryptamine has been proposed as a mechanism ${ }^{15}$ and might plausibly also account for the development of hyperacusis in migraine and depression. It has also been described following focal cortical lesions ${ }^{16} 17$ although few imaging studies have been conducted. ${ }^{18}$

In this study, we addressed neuroanatomical associations of tinnitus and hyperacusis in patients with semantic dementia (SemD) using voxel based morphometry (VBM). SemD is a canonical language led dementia (progressive aphasia) syndrome in the frontotemporal lobar degeneration spectrum associated with focal asymmetric atrophy, particularly affecting the anteroinferior temporal lobes. ${ }^{19}$ SemD is characterised by progressive erosion of semantic memory, typically first manifesting as impaired verbal comprehension with development of non-verbal semantic and behavioural features as the process spreads within and between the temporal lobes and adjacent inferior frontal lobes. ${ }^{20}$ Our motivation for undertaking this study was our clinical impression that patients with SemD commonly report tinnitus and hyperacusis. This clinical impression has some prima facie support: alterations of auditory perception and auditory emotional responses appear to be significant in $\mathrm{SemD}^{21}$ and SemD involves a number of cortical and limbic areas previously implicated in the pathogenesis of tinnitus. $^{5} 622$ We hypothesised that damage 
Table 1 Neuroanatomical group studies of tinnitus and hyperacusis

\begin{tabular}{|c|c|c|c|c|c|c|}
\hline Study & Subjects & Methods & Modality & Anatomical association & Activity & Grey matter \\
\hline Shulman et al $1995^{41}$ & $\begin{array}{l}2 \mathrm{TI} \\
5 \text { Controls }\end{array}$ & Resting state & SPECT & Bilateral hippocampi, amygdala, PFC & Decreased & \\
\hline Arnold et al $1996^{11}$ & $\begin{array}{l}11 \mathrm{Tl} \\
12 \text { Controls }\end{array}$ & Resting state & PET & Primary AC & Increased & \\
\hline Lockwood et al $1998^{10}$ & $\begin{array}{l}4 \mathrm{TI} \\
2 \mathrm{TI}\end{array}$ & $\begin{array}{l}\text { Facial movement } \\
\text { Movement inc TI }\end{array}$ & PET & $\begin{array}{l}\text { L primary /non-primary AC } \\
\text { L MGN }\end{array}$ & $\begin{array}{l}\text { Decreased } \\
\text { Increased }\end{array}$ & \\
\hline Mirz et al $1999^{27}$ & $\begin{array}{l}12 \mathrm{TI} \\
10 \mathrm{TI}\end{array}$ & $\begin{array}{l}\text { Noise masking } \\
\text { Lignocaine }\end{array}$ & PET & $\begin{array}{l}\text { Primary } \mathrm{AC} \\
\mathrm{R} \text { prefrontal/parietal }\end{array}$ & $\begin{array}{l}\text { Increased } \\
\text { Decreased }\end{array}$ & \\
\hline Melcher et al $2000^{12}$ & $\begin{array}{l}7 \mathrm{TI} \\
6 \text { Controls }\end{array}$ & $\begin{array}{l}\text { Noise masking; } \\
\text { Residual inhibition }\end{array}$ & fMRI & $\mathrm{R}$ inferior colliculus & Decreased contra to $\mathrm{TI}$ & \\
\hline Reyes et al $2002^{28}$ & $\begin{array}{l}9 \mathrm{TI} \\
9 \text { Controls }\end{array}$ & Lignocaine & PET & $\begin{array}{l}\mathrm{R} \mathrm{AC} \\
\mathrm{ACC} \text {, thalamus }\end{array}$ & $\begin{array}{l}\text { Decreased } \\
\text { Increased }\end{array}$ & \\
\hline Osaki et al $2005^{29}$ & $\begin{array}{l}3 \text { TI } \\
6 \text { Controls }\end{array}$ & Residual inhibition & PET & $\mathrm{R}$ anterior/middle temporal lobe & Increased & \\
\hline Mühlau et al $2006^{9}$ & $\begin{array}{l}28 \mathrm{Tl} \\
28 \text { Controls }\end{array}$ & VBM & vMRI & $\begin{array}{l}\text { Subcallosal area } \\
\text { R MGN }\end{array}$ & - & $\begin{array}{l}\text { Decreased } \\
\text { Increased }\end{array}$ \\
\hline Plewnia et al $2007^{30}$ & $9 \mathrm{TI}$ & Lignocaine & PET & $\begin{array}{l}\text { R temperoparietal junc } \\
\mathrm{L} \text { mid/inf temporal lobe, L PCC }\end{array}$ & Increased & \\
\hline Smits et al $2007^{7}$ & $\begin{array}{l}35 \mathrm{Tl} \text { (Lat) } \\
10 \text { Bilateral }\end{array}$ & Bilateral music & fMRI & $\begin{array}{l}\text { IC, MGN, association } A C \\
L A C\end{array}$ & $\begin{array}{l}\text { Decreased contra to } \mathrm{TI} \\
\text { Decreased }\end{array}$ & \\
\hline Hwang et al $2009^{18}$ & $\begin{array}{l}3 \mathrm{HA} \\
3 \text { Controls }\end{array}$ & Bilateral noise & fMRI & $\begin{array}{l}\text { Bilateral PFC, OFC, PH, } \\
\text { parieto-occipital junction, L PCC, DL-PFC }\end{array}$ & Increased & \\
\hline Landgrebe et al $2009^{8}$ & $\begin{array}{l}20 \mathrm{TI}+8 \text { Lat } \\
28 \text { Controls }\end{array}$ & VBM & vMRI & $\begin{array}{l}\mathrm{R} \text { inferior colliculus, } \\
\mathrm{L} \text { hippocampus }\end{array}$ & & Decreased \\
\hline Schlee et al $2009^{35}$ & $\begin{array}{l}23 \mathrm{TI} \\
24 \text { Controls }\end{array}$ & Resting state & MEG & $\begin{array}{l}\mathrm{R}>\mathrm{L} \text { parieto-occiptal, bilat PFC, } \\
\mathrm{R} \text { OFC }\end{array}$ & $\begin{array}{l}\text { Increased (outflow) } \\
\text { Decreased (outflow) }\end{array}$ & \\
\hline Present study & $\begin{array}{l}7 \mathrm{TI} \\
7 \mathrm{HA}\end{array}$ & VBM & vMRI & $\begin{array}{l}\text { R STG/STS } \\
\text { Bilateral OFC } \\
\text { L MGN }\end{array}$ & & $\begin{array}{l}\text { Increased } \\
\text { Decreased } \\
\text { Decreased }\end{array}$ \\
\hline
\end{tabular}

$\mathrm{AC}$, auditory cortex; $\mathrm{ACC}$, anterior cingulate cortex; DL-PFC, dorsolateral prefrontal cortex; fMRI, functional MRI; HA, hyperacusis; IC, inferior colliculus; L AC, left auditory cortex; Lat, unilateral MEG, magnetoencephalography; MGN, medial geniculate nucleus; OFC, orbitofrontal cortex; PCC, posterior cingulate cortex; PET, positron emission tomography; PFC, prefrontal cortex; PH, parahippocampus; SPECT, single photon emission CT; STG/STS, superior temporal gyrus/sulcus; TI, tinnitus; VBM, voxel based morphometry; vMRI, volumetric MRI.

involving such areas in SemD would make these patients more liable to develop tinnitus and hyperacusis. More specifically, we hypothesised that, compared with patients with SemD who had not developed auditory symptoms, patients with SemD who had auditory symptoms would exhibit relatively greater involvement of cortical and subcortical auditory pathways and extratemporal areas concerned with the evaluation of sensory stimuli (in particular, the inferior frontal cortex).

\section{METHODS \\ Subjects}

The case notes of patients with a diagnosis of SemD attending our specialist cognitive clinic over an 18 year period were retrospectively reviewed and all cases with volumetric brain MRI were included in the study. If the case notes clearly documented whether or not the patient was experiencing tinnitus or hyperacusis, this was recorded; if there was no record of any auditory disturbance in the case notes, patients were classified as having no auditory symptoms. Forty-three patients (24 men, mean (SD) age 64.4 (7.6) years) were ascertained. All patients presented with a clinically typical syndrome of SemD led by loss of vocabulary and evidence of asymmetric (predominantly leftsided) temporal lobe atrophy. Fourteen patients (32\% of the SemD cohort) had complained of chronic auditory symptoms (predominantly tinnitus in seven cases, predominantly hyperacusis in seven cases). In order to corroborate the retrospective auditory history, we attempted to contact patients' carers by telephone to administer modified versions of the National Study of Hearing questionnaire, the THI and the Hyperacusis Questionnaire. ${ }^{43}$ Carers (the patients' spouses) also completed the questionnaires in respect of their own auditory history.

The study was conducted in accordance with the Declaration of Helsinki and ethics approval was obtained from the joint research ethics committees of the National Hospital for Neurology and Neurosurgery and the Institute of Neurology.

\section{MRI acquisition}

Each patient had brain MRI on a $1.5 \mathrm{~T}$ scanner using a standard quadrature head coil. T1 weighted volumetric images were obtained with a $24 \mathrm{~cm}$ field of view and $256 \times 256$ matrix to provide 124 contiguous, $1.5 \mathrm{~mm}$ thick slices in the coronal plane (echo time $5 \mathrm{~ms}$, repetition time $12 \mathrm{~ms}$, inversion time $650 \mathrm{~ms}$ ).

\section{Image analysis}

VBM of brain images was performed using the DARTEL toolbox of SPM5 (http://www.fil.ion.ucl.ac.uk/spm) running under Matlab 7.0 (Mathworks, Sherborn, Massachusetts, USA), and normalisation, segmentation, modulation and smoothing of grey and white matter images were performed using default parameter settings. ${ }^{24}$ In order to adjust for individual differences in global grey matter volume, total intracranial volume (TIV) was calculated for each subject by summing grey matter, white matter and CSF volumes following segmentation of all three tissue classes. Linear regression models were used to examine regional grey matter volumes correlated with the presence or absence of symptoms in a combined group analysis. Voxel intensity, $v$, was modelled as a function of symptomatic status (present/absent) across the group. Subject age, gender and TIV were included as nuisance covariates: $v=\beta_{1}$ symptom status + $\beta_{2}$ age $+\beta_{3}$ gender $+\beta_{4}$ TIV $+\mu+\varepsilon$ (where $\mu$ is a constant and $\varepsilon$ is error). Grey matter associations were evaluated using a common design matrix which included all symptomatic patients and in separate design matrices including patients with tinnitus and patients with hyperacusis; in each design, the comparator group was patients without auditory symptoms. Analyses were performed over voxels inside a 'consensus mask'25 which included all voxels with intensity $>0.1$ in $>70 \%$ of subjects.

Statistical parametric maps of regional grey matter intensity associated with symptomatic status were examined at a voxel wise threshold of $p<0.05$ (with a cluster extent threshold of 
30 voxels) after small volume correction in accordance with the prior anatomical hypotheses. Small anatomical volumes were created in MRIcron: these volumes comprised each temporal lobe (a region extending from the anterior temporal pole posteriorly to the parieto-occipital junction and from the superior temporal gyrus medially to the medial border of Heschl's gyrus, including auditory cortices and mesial temporal limbic structures), the upper brainstem (a region extending from the upper pons rostrally to the mid thalamus and from the ventral border of the pons posteriorly to the mid cerebellar peduncles, including both inferior colliculi and medial geniculate nuclei) and bilateral orbitofrontal cortices (a region extending from the gyrus rectus medially to the lateral orbital gyrus and from the frontal pole to the border of the posterior orbital gyrus and insula). In order to assess any grey matter associations of tinnitus or hyperacusis in relation to the overall distribution of disease related brain damage, in a separate VBM subanalysis we derived a map of SemD related brain atrophy by comparing $M R$ images from the SemD cohort with $M R$ images (obtained using the same scanner and acquisition parameters) from a group of 20 age and gender matched healthy control subjects (mean age 65.2 years (7.3); eight women; TIV $1620 \mathrm{ml}(152))$. The atrophy map was thresholded at $\mathrm{p}<0.05$ after false discovery rate correction over the whole brain.

\section{RESULTS}

\section{Clinical characteristics}

Patient characteristics are summarised in table 2. Of the 14 patients reporting auditory symptoms, five reported tinnitus alone, two both tinnitus and hyperacusis and seven hyperacusis alone. All of these individuals had persistent (chronic) symptoms consistent with significant tinnitus or hyperacusis present for at least 1 year prior to their MRI scan, and MRI in each case was acquired while the patient was symptomatic. Patients in the subgroups with and without auditory symptoms had a similar mean clinical disease duration (symptomatic $=5.07$ years $( \pm 2.87)$; non-symptomatic $=4.62$ years $( \pm 2.07)(p=0.56))$. Four symptomatic and six non-symptomatic individuals had additional non-verbal symptoms (behavioural change or prosopagnosia) at presentation. Naming performance (an index of semantic impairment-ie, SemD severity) was severely impaired in both subgroups, and the subgroups had similar profiles of executive impairment. Symptom questionnaires were administered to eight carers (two for patients with tinnitus, six for patients with hyperacusis; see table 2). Using the National Study of Hearing questionnaire, no hearing deficit was predicted for any of the patients reporting hyperacusis alone; a hearing deficit equivalent to whispered speech (ie, $9 \mathrm{~dB}^{2}$ ) was predicted for both patients with tinnitus whose carers were interviewed, and based on the THI tinnitus was graded to have been moderately severe in both cases. One of these patients had a known history of congenital sensorineural hearing loss but complained of tinnitus only after the onset of SemD. One patient reported onset of tinnitus 3 years prior to onset of the language syndrome. All patients with questionnaire data reported hyperacusis: this was given a mean scaled distress score $>28$ on the modified Hyperacusis Questionnaire, indicating significant auditory hypersensitivity. ${ }^{23}$ In other cases where questionnaire data were not available, a qualitative review of the clinical history indicated that auditory symptoms were persistent, intrusive or distressing, with an impact on the patient's quality of life, suggesting that these symptoms were at least moderately severe. Only one of eight carers themselves reported hyperacusis (graded as mild); none reported tinnitus.

\section{VBM data}

Associations between regional grey matter intensity and auditory symptoms were identified within the SemD cohort; statistical parametric maps of regional grey matter associations are shown in figure 1. Compared with the patient subgroup without auditory symptoms, the patient subgroup with auditory symptoms (considering tinnitus and hyperacusis together) had increased grey matter in the right posterior superior temporal gyrus and sulcus and reduced grey matter in bilateral orbitofrontal cortices (both $\mathrm{p}<0.05$ after small volume correction). No grey matter differences were observed in early auditory

Table 2 Patient characteristics

\begin{tabular}{|c|c|c|c|c|}
\hline & Tinnitus & Hyperacusis & Nil auditory & All \\
\hline No of patients & 7 & 7 & 29 & 43 \\
\hline Age (years) & $61.5( \pm 5.1)$ & $60.2( \pm 5.9)$ & $66( \pm 7.9)$ & $64.4( \pm 7.6)$ \\
\hline Gender (M:F) & $4: 3$ & $5: 2$ & $15: 14$ & $24: 19$ \\
\hline Symptom duration to MRI (years) & $5.1( \pm 3.0)$ & $5( \pm 3.0)$ & $4.62( \pm 2.1)$ & $4.8( \pm 2.3)$ \\
\hline \multicolumn{5}{|l|}{ Naming } \\
\hline GNT & $0(n=1)$ & $0.5(n=2)$ & $0.5(n=8)$ & $0.5(n=11)$ \\
\hline ONT & $5.8(n=6)$ & $3.2(n=5)$ & $6.6(n=21)$ & $5.78(n=32)$ \\
\hline \multicolumn{5}{|l|}{ Executive function $₫$} \\
\hline$>10$ th centile & 3 & 4 & 15 & 22 \\
\hline $5-10$ th centile & 1 & 0 & 3 & 4 \\
\hline$<5$ th centile & 1 & 2 & 4 & 7 \\
\hline Other non-verbal symptoms* & 2 & 2 & 6 & 10 \\
\hline Hearing deficit (NSH) & $\mathrm{N}=2$ & 0 & N/A & $\mathrm{N} / \mathrm{A}$ \\
\hline THI scaled score & $42 / 100(n=2) \dagger$ & $\mathrm{N} / \mathrm{A}$ & $\mathrm{N} / \mathrm{A}$ & $\mathrm{N} / \mathrm{A}$ \\
\hline HO scaled score $\ddagger$ & $28.9 / 42( \pm 11.1) ;(n=2 \S)$ & $30.6 / 42( \pm 5.6) ;(n=6)$ & $\mathrm{N} / \mathrm{A}$ & $\mathrm{N} / \mathrm{A}$ \\
\hline \multicolumn{5}{|c|}{$\begin{array}{l}\text { *Behavioural change or prosopagnosia at presentation. } \\
\text { †Moderately severe. } \\
\text { †Modified questionnaire from which scaled score devised to range from } 0 \text { (no symptom distress) to } 42 \text { (maximum distress) and score } \\
>28=\text { significant auditory hypersensitivity. } \\
\text { §Both of these patients also reported hyperacusis. } \\
\text { \Standard tests of executive function (available for } 33 \text { of } 43 \text { patients) included the Weigl Card Sorting Test, the Wisconsin Card } \\
\text { Sorting Test, the Trail Making Test Part B and the Stroop Task; best performance on any executive test is recorded. } \\
\text { GNT, Graded Naming Test; HQ, Hyperacusis Questionnaire; N/A, not available; NSH, National Study of Hearing Questionnaire; ONT, } \\
\text { Oldfield Naming Test; THI, Tinnitus Handicap Inventory. }\end{array}$} \\
\hline
\end{tabular}


Figure 1 Statistical parametric maps of grey matter differences associated with auditory symptom status have been rendered on the mean T1weighted normalised DARTEL brain image for the SemD cohort. For display purposes, all maps are thresholded at $p<0.001$ uncorrected and show clusters $>30$ voxels in size; for all regions shown, local maxima were significant at $p<0.05$ corrected for multiple comparisons over the pre-specified anatomical volume of interest (see text). T score is coded on the colour bar (lower right). L, left; $R$, right; *axial sections have been tilted to run along the superior temporal sulcus (showing auditory association cortex).

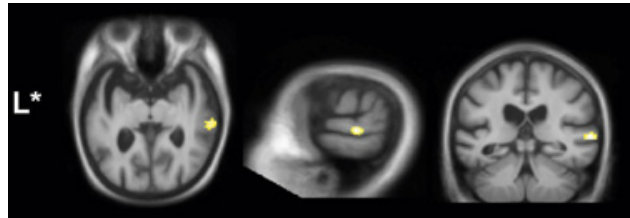

Symptomatic $>$ Non-symptomatic

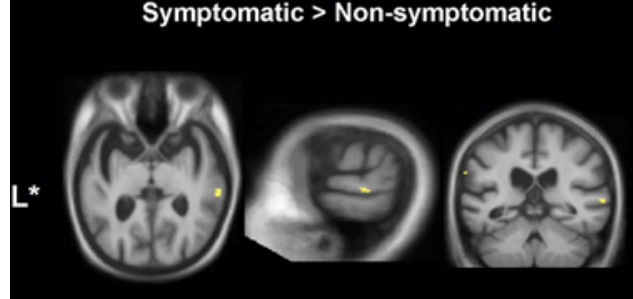

Tinnitus $>$ Non-symptomatic

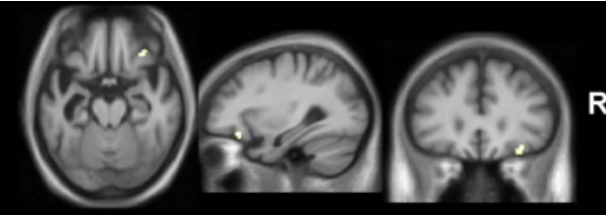

Non-symptomatic > Symptomatic

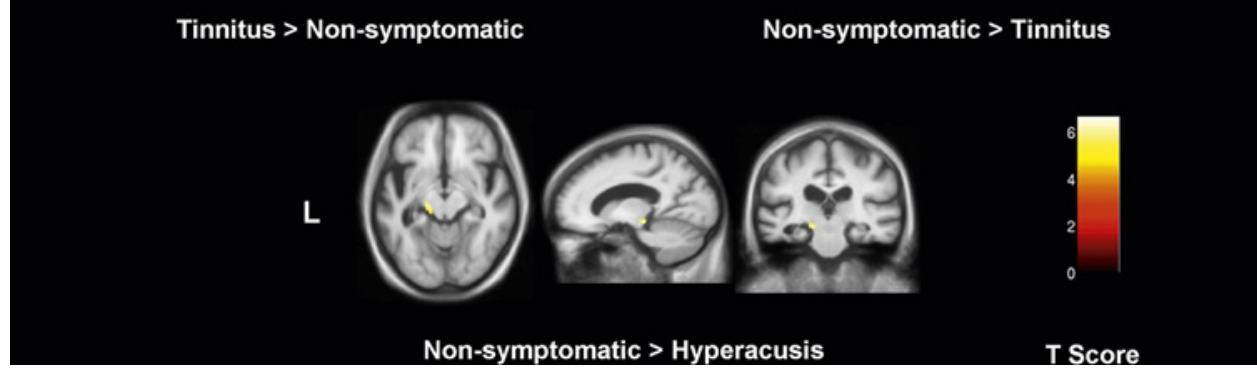

Non-symptomatic $>$ Hyperacusis cortical regions in the superior temporal plane. The subgroup with predominant tinnitus showed a similar neuroanatomical profile. Compared with the patient subgroup without auditory symptoms, the subgroup with hyperacusis only had reduced grey matter in the left medial geniculate nucleus $(p<0.05$ after small volume correction). All grey matter areas identified in the disease subgroup contrasts were located within the region of disease associated atrophy in the contrast between the SemD and healthy control groups, indicating that any regional grey matter increase was relative rather than absolute.

\section{DISCUSSION}

Here we present evidence that symptoms of abnormal auditory perception are significant in SemD and have specific neuroanatomical substrates. The overall prevalence of auditory symptoms in our SemD cohort (14/32 cases, 32\%) was substantially higher than the estimated combined prevalence of these symptoms among older adults in the general population $(<20 \%)$; while considering tinnitus alone (for which more detailed population information is available), $7 / 32$ patients $(16 \%)$ in the SemD cohort reported moderate or severe chronic tinnitus compared with an estimated prevalence of moderately severe tinnitus of $4-6 \%$ of older adults in the general population. ${ }^{2} 26$ As patients' spouses reported a low frequency of auditory symptoms, it is unlikely the apparently increased prevalence of these symptoms in our SemD cohort was attributable to environmental or other extraneous factors. The neuroanatomical findings here implicate a distributed cortico-subcortical network, including both auditory and non-auditory areas, in the pathogenesis of auditory symptoms in SemD: atrophy of the orbitofrontal cortex was associated with tinnitus; atrophy of the medial geniculate nucleus (auditory thalamus) was associated with hyperacusis; while relative preservation of grey matter in the posterior superior temporal lobe was associated with both tinnitus and hyperacusis. These findings are broadly consistent with previous work in other populations with chronic tinnitus and hyperacusis. ${ }^{7-12} 1618$ 27-32

As peripheral hearing was not directly assessed in our SemD cohort, we cannot be certain to what extent peripheral deficits contributed to the pathogenesis of auditory symptoms in these patients; however, the neuroanatomical data here suggest a role for central mechanisms in the generation of abnormal auditory percepts in the context of brain disease. We also note that Heschl's gyrus (the seat of primary auditory cortex) was not implicated in our study, in contrast with previous work on tinnitus in association with peripheral deafness ${ }^{33}$ but consistent with recent work indicating the clinical and anatomical dissociability of hearing loss and tinnitus. ${ }^{34}$ Our findings illustrate the role of distributed brain networks in producing abnormal auditory percepts, with or without a facilitating effect from peripheral hearing loss. ${ }^{18} 35$ The present findings further suggest that the limbic or paralimbic (orbitofrontal) cortex is involved in the pathogenesis of tinnitus and hyperacusis, presumably conditioning the powerful emotional response to the symptoms that most sufferers describe. ${ }^{10} 22{ }^{35-37}$ It is of interest that we found both regional loss and relative preservation of grey matter in association with altered auditory perception: this implies that tinnitus and hyperacusis are not simply a consequence of net loss or gain of cortical function but may instead result from altered coupling within a larger cortical-subcortical neural network. This suggestion is in line with current models of the pathogenesis of tinnitus and hyperacusis that emphasise complex interactions between sensory insufficiency, sustained hyperexcitability within central auditory pathways and abnormal modulation by a limbic 'gating system'. 22 The area of relative grey matter increase in the posterior superior temporal gyrus and sulcus identified here contains the association auditory cortex and has been shown previously to be abnormally activated in patients with chronic tinnitus. ${ }^{7}$ The use of a structural imaging modality here does not address the possibility of more widespread functional alterations involving structurally unaffected brain areas in the auditory cortex and beyond.

Our findings corroborate other emerging evidence for central auditory dysfunction in common degenerative dementias, including Alzheimer's disease ${ }^{38}$ and diseases in the frontotemporal lobar degeneration spectrum. ${ }^{39}$ It is tempting to speculate that SemD may particularly predispose to the development of central auditory disorders because the tissue damage 
in SemD selectively targets a number of areas in the temporal and adjacent frontal lobes that are likely to be critical for the perception, comprehension and affective response to sound. This suggestion would be consistent with previous clinical observations that patients with focal temporal lobe atrophy may have heightened sensitivity to sensory stimuli of various kinds. ${ }^{40}$ Limitations of this study include its retrospective nature, the relatively small number of cases and reliance on a single (structural) imaging modality. Tinnitus and hyperacusis are not unitary symptoms and the phenomenological characteristics of these percepts in relation to peripheral and central auditory function are likely to be informative. Future work should address these issues in prospective, longitudinal studies using multimodal clinical, electrophysiological, structural and functional imaging techniques in larger cohorts of patients with SemD and other neurodegenerative conditions.

Acknowledgements We are grateful to all of our subjects for their participation.

Funding This work was undertaken at UCLH/UCL who received a proportion of funding from the Department of Health's NIHR Biomedical Research Centres funding scheme. The Dementia Research Centre is an Alzheimer's Research UK Co-ordinating Centre. This work was also funded by the Medical Research Council UK. CJM is supported by an MRC programme grant. MNR and NCF are NIHR senior investigators. JDW is supported by a Wellcome Trust Senior Clinical Fellowship.

Competing interests None.

Ethics approval Ethics approval was provided by the joint research ethics committees of the National Hospital for Neurology and Neurosurgery and the Institute of Neurology.

Provenance and peer review Not commissioned; externally peer reviewed.

\section{REFERENCES}

1. Andersson G, Lindvall N, Hursti T, et al. Hypersensitivity to sound (hyperacusis): a prevalence study conducted via the Internet and post. Int J Audiol 2002;41:545-54.

2. Davis AC. The prevalence of hearing impairment and reported hearing disability among adults in Great Britain. Int J Epidemiol 1989;18:911-17.

3. Demeester K, van Wieringen A, Hendrickx JJ, et al. Prevalence of tinnitus and audiometric shape. B-ENT 2007; (Suppl 7):37-49.

4. Newman CW, Jacobson GP, Spitzer JB. Development of the Tinnitus Handicap Inventory. Arch Otolaryngol Head Neck Surg 1996;122:143-8.

5. Eggermont J, Roberts L. The neuroscience of tinnitus. Trends Neurosci 2004;27:676-82.

6. Shulman A, Goldstein B, Strashun AM. Final common pathway for tinnitus: theoretical and clinical implications of neuroanatomical substrates. Int Tinnitus $J$ 2009;15:5-50.

7. Smits M, Kovacs S, Ridder D, et al. Lateralization of functional magnetic resonance imaging (fMRI) activation in the auditory pathway of patients with lateralized tinnitus. Neuroradiology 2007:49:669-79.

8. Landgrebe $\mathbf{M}$, Langguth $B$, Rosengarth $\mathrm{K}$, et al. Structural brain changes in tinnitus: grey matter decrease in auditory and non-auditory brain areas. Neuroimage 2009:46:213-18.

9. Mühlau M, Rauschecker JP, Oestreicher E, et al. Structural brain changes in tinnitus. Cereb Cortex 2006;16:1283-8.

10. Lockwood AH, Salvi RJ, Coad ML, et al. The functional neuroanatomy of tinnitus: evidence for limbic system links and neural plasticity. Neurology 1998;50:114-20.

11. Arnold W, Bartenstein P, Oestreicher E, et al. Focal metabolic activation in the predominant left auditory cortex in patients suffering from tinnitus: a PET study with [18F]deoxyglucose. ORL J Otorhinolaryngol Relat Spec 1996;58:195-9.
12. Melcher JR, Sigalovsky IS, Guinan JJ, et al. Lateralized tinnitus studied with functional magnetic resonance imaging: abnormal inferior colliculus activation. J Neurophysiol 2000;83:1058-72.

13. Kelman L, Tanis D. The relationship between migraine pain and other associated symptoms. Cephalalgia 2006;26:548-53.

14. Gothelf D, Farber N, Raveh E, et al. Hyperacusis in Williams syndrome: characteristics and associated neuroaudiologic abnormalities. Neurology 2006;66:390-5.

15. Marriage J, Barnes NM. Is central hyperacusis a symptom of 5-hydroxytryptamine (5-HT) dysfunction? J Laryngol Otol 1995:109:915-21.

16. Fukutake T, Hattori T. Auditory illusions caused by a small lesion in the right media geniculate body. Neurology 1998;51:1469-71.

17. Weber H, Pfadenhauer K, Stöhr M, et al. Central hyperacusis with phonophobia in multiple sclerosis. Mult Scler 2002;8:505-9.

18. Hwang J, Chou P, Wu C, et al. Brain activation in patients with idiopathic hyperacusis. Am J Otolaryngol 2009;30:432-4.

19. Hodges JR, Patterson K. Semantic dementia: a unique clinicopathological syndrome. Lancet Neurol 2007:6:1004-14.

20. Rohrer JD, Warren JD, Modat M, et al. Patterns of cortical thinning in the language variants of frontotemporal lobar degeneration. Neurology 2009;72:1562-9.

21. Omar R, Hailstone JC, Warren JE, et al. The cognitive organization of music knowledge: a clinical analysis. Brain 2010;133:1200-13.

22. Rauschecker JP, Leaver AM, Mühlau M. Tuning out the noise: limbic-auditory interactions in tinnitus. Neuron 2010;66:819-26.

23. Khalfa S, Dubal $S$, Veuillet $E$, et al. Psychometric normalization of a hyperacusis questionnaire. ORL 2002;64:436-42.

24. Ridgway GR, Henley SMD, Rohrer JD, et al. Ten simple rules for reporting voxel-based morphometry studies. Neuroimage 2008;40:1429-35.

25. Ridgway GR, Omar R, Ourselin $\mathrm{S}$, et al. Issues with threshold masking in voxel-based morphometry of atrophied brains. Neuroimage 2009;44:99-111.

26. Gudex C, Skellgaard PH, West T, et al. Effectiveness of a tinnitus management programme: a 2-year follow-up study. BMC Ear Nose Throat Disord 2009;9:6.

27. Mirz F, Pedersen B, Ishizu K, et al. Positron emission tomography of cortical centers of tinnitus. Hear Res 1999;134:133-44.

28. Reyes SA, Salvi RJ, Burkard RF, et al. Brain imaging of the effects of lidocaine on tinnitus. Hear Res 2002;171:43-50.

29. Osaki Y, Nishimura H, Takasawa M, et al. Neural mechanism of residual inhibition of tinnitus in cochlear implant users. Neuroreport 2005:16:1625-8.

30. Plewnia C, Reimold M, Najib A, et al. Dose-dependent attenuation of auditory phantom perception (tinnitus) by PET-guided repetitive transcranial magnetic stimulation. Hum Brain Mapp 2007;28:238-46.

31. Lanting $\mathbf{C P}$, de Kleine E, van Dijk P. Neural activity underlying tinnitus generation: results from PET and fMRI. Hear Res 2009;255:1-13.

32. Shulman A, Goldstein B, Strashun AM. Central nervous system neurodegeneration and tinnitus: a clinical experience. Part II: translational neurovascular theory of neurodegenerative CNS disease and tinnitus. Int Tinnitus J 2008;14:43-51.

33. Schneider P, Andermann M, Wengenroth $\mathrm{M}$, et al. Reduced volume of Heschl's gyrus in tinnitus. Neuroimage 2009;45:927-39.

34. Husain FT, Medina RE, Davis CW, et al. Neuroanatomical changes due to hearing loss and chronic tinnitus: a combined VBM and DTI study. Brain Res 2011:1369:74-88.

35. Schlee W, Mueller N, Hartmann T, et al. Mapping cortical hubs in tinnitus. BMC Biol 2009; 7:80.

36. Vanneste $\mathbf{S}$, Plazier M, der Loo EV, et al. The neural correlates of tinnitus-related distress. Neurolmage. 2010;52:470-80.

37. Barbas H. Flow of information for emotions through temporal and orbitofrontal pathways. J Anat 2007:211:237-49.

38. Gates GA, Anderson ML, Feeney MP, et al. Central auditory dysfunction in older persons with memory impairment or Alzheimer dementia. Arch Otolaryngol Head Neck Surg 2008;134:771-7.

39. Goll JC, Crutch SJ, Loo JHY, et al. Non-verbal sound processing in the primary progressive aphasias. Brain 2010:133:272-85.

40. Edwards-Lee T, Miller BL, Benson DF, et al. The temporal variant of frontotempora dementia. Brain 1997;120:1027-40.

41. Shulman A, Strashun AM, Afriyie M, et al. SPECT Imaging of Brain and Tinnitus-Neurotologic/Neurologic Implications. Int Tinnitus J 1995;1:13-29. 